Mathematical Modelling and Analysis

Volume 19 Number 3, June 2014, 430-442

http://dx.doi.org/10.3846/13926292.2014.929057

(C) Vilnius Gediminas Technical University, 2014
Publisher: Taylor\&Francis and VGTU

http://www.tandfonline.com/TMMA

Print ISSN: 1392-6292

Online ISSN: 1648-3510

\title{
A Truncation Method Based on Hermite Expansion for Unknown Source in Space Fractional Diffusion Equation
}

\author{
Zhenyu Zhao $^{a}$, Ou Xie ${ }^{a}$, Lei You ${ }^{a}$ and Zehong Meng ${ }^{b}$ \\ ${ }^{a}$ College of Science, Guangdong Ocean University \\ 524088 Zhanjiang, China \\ ${ }^{b}$ School of Mathematics and Statistics, Zhejiang University of Finance and \\ Economics \\ 310018 Hangzhou, China \\ E-mail(corresp.): ouxiesubmit@163.com \\ E-mail: dongdongzb@gmail.com
}

Received August 3, 2013; revised May 23, 2014; published online June 1, 2014

\begin{abstract}
In this paper we consider the problem for identifying an unknown steady source in a space fractional diffusion equation. A truncation method based on a Hermite function expansion is proposed, and the regularization parameter is chosen by a discrepancy principle. An error estimate between the exact solution and its approximation is given. A numerical implementation is discussed and corresponding results are presented to verify the effectiveness of the method.
\end{abstract}

Keywords: ill-posed problem, unknown source, truncation method, Hermite functions expansion, discrepancy principle.

AMS Subject Classification: $65 \mathrm{~F} 22$.

\section{Introduction}

In recent years, fractional calculus and fractional differential equations have attracted great attention in the scientific and engineering community. One of the most successful applications of fractional calculus and fractional differential equations is to effectively characterize the anomalous diffusion $[11,12]$. The anomalous diffusion is described by the nonlinear growth of the mean square displacement $x(t)$ of a diffusion particle over time $t:\left\langle x^{2}(t)\right\rangle \sim \kappa_{\alpha} t^{\alpha}$, where $\kappa_{\alpha}$ is the diffusion coefficient, and $\alpha$ is the anomalous diffusion exponent. For different $\alpha$, the anomalous diffusion is classified into subdiffusion $(0<\alpha<1)$, normal diffusion $(\alpha=1)$, superdiffusion $(\alpha>1)$, and ballistic diffusion $(\alpha=2)$ $[11,18]$.

The space fractional diffusion equation we consider in this paper can describe the probability distribution of the particles having superdiffusion [18] 


$$
\frac{\partial u(x, t)}{\partial t}=-\frac{\partial J_{p, q}^{\alpha}(x, t)}{\partial x}+f(x)
$$

where

$$
J_{p, q}^{\alpha}(x, t)=-\kappa_{\alpha}\left\{p_{-\infty} D_{x}^{\alpha-1}-q_{x} D_{\infty}^{\alpha-1}\right\} u
$$

with $\kappa_{\alpha}>0$, and $p+q=1$ for $p, q \geq 0$. The fractional derivatives ${ }_{-\infty} D_{x}^{\alpha}$ and ${ }_{x} D_{\infty}^{\alpha}$ are left and right Riemann-Liouville fractional derivatives of order $\alpha(1<\alpha<2)$ defined by

$$
{ }_{-\infty} D_{x}^{\alpha} u(x, t)=\frac{1}{\Gamma(2-\alpha)} \frac{d^{2}}{d x^{2}} \int_{-\infty}^{x}(x-\varsigma)^{1-\alpha} u(\varsigma, t) d \varsigma
$$

and

$$
{ }_{x} D_{\infty}^{\alpha} u(x, t)=\frac{(-1)^{\lceil\alpha\rceil}}{\Gamma(2-\alpha)} \frac{d^{2}}{d x^{2}} \int_{x}^{\infty}(\varsigma-x)^{1-\alpha} u(\varsigma, t) d \varsigma,
$$

such that $\lceil\alpha\rceil$ is the smallest integer no less than $\alpha$ (for $1<\alpha<2$, we have $\lceil\alpha\rceil=2)$.

Our goal is to identify the source term $f(x)$ in the equation (1.1) with the boundary conditions

$$
u(x, 0)=0, \quad u(x, T)=g(x)
$$

that is, to determine the source $f(x)$ in (1.1) from some observed boundary data in (1.2): $g \rightarrow f$. This problem is called the inverse source problem [9], and it is one of the most important problems in many branches of engineering sciences. Inverse source problems are well known to be ill posed and the data $g(x)$ usually contains error. Thus, the numerical simulation of these problems is very difficult and some special regularization is required.

Some results on the inverse problem of time fractional diffusion equations have been reported $[3,4,10,13]$. However, there is limited work on space fractional diffusion equation $[14,18,21]$. There are some discussions on special cases of the inverse problem (1.1), namely $\alpha \rightarrow 2$, which refers to the sideways heat equation $[2,5,6,19,24]$. In the present paper, as an alternative way of dealing with the problem, we introduce a truncation method based on a Hermite expansion. The method is a very simple and effective method for solving some ill-posed problems and it has been successfully applied to numerical differentiation [23] and numerical analytic continuation [22]. We choose a regularization strategy based on a discrepancy principle explained in [20]. The theoretical analysis shows that the smoother the genuine solution, the higher the convergence rate of the numerical solution by our method.

This paper is organized as follows. Some preliminary materials are introduced in Section 2. In Section 3, the present method and corresponding convergence results are given. Some numerical tests are given in Section 4 to show the effectiveness of the new method. 


\section{Formulation of Problem and Solution}

Let $\hat{h}$ denote the Fourier transform of $h \in L^{2}(\mathbb{R})$ defined by

$$
\hat{h}(\xi)=\mathcal{F}[h(x)]=\frac{1}{\sqrt{2 \pi}} \int_{-\infty}^{\infty} h(x) e^{-\mathrm{i} \xi x} d x
$$

and $\|\cdot\|_{s}$ denotes the norm in Sobolev space $H^{s}(\mathbb{R})(s \geq 0)$ defined by

$$
\|h\|_{s}:=\left(\int_{\infty}^{\infty}\left(1+\xi^{2}\right)^{s}|\hat{h}(\xi)|^{2} d \xi\right)^{1 / 2} .
$$

When $s=0,\|\cdot\|_{0}=:\|\cdot\|$ denotes the $L^{2}(\mathbb{R})$ norm. It is well known that the Fourier transform of ${ }_{-\infty} D_{x}^{\alpha}$ and ${ }_{x} D_{\infty}^{\alpha}$ can be given as [15]

$\mathcal{F}\left\{{ }_{-\infty} D_{x}^{\alpha} u(x, t)\right\}=(i \xi)^{\alpha} \hat{u}(\xi, t), \quad \mathcal{F}\left\{{ }_{x} D_{\infty}^{\alpha} u(x, t)\right\}=(-i \xi)^{\alpha} \hat{u}(\xi, t), \quad \xi \in \mathbb{R}$.

Then we have

$$
\mathcal{F}\left\{J_{p, q}^{\alpha}(x, t)\right\}=\left[p(i \xi)^{\alpha}+q(-i \xi)^{\alpha}\right] \hat{u}(\xi, t), \quad \xi \in \mathbb{R} .
$$

According to the definition of the complex power function, the principal value of the Fourier coefficient in (2.1) is [18]

$$
p(i \xi)^{\alpha}+q(-i \xi)^{\alpha}=\left[\cos \left(\frac{\pi \alpha}{2}\right)+i(p-q) \operatorname{sgn}(\xi) \sin \left(\frac{\pi \alpha}{2}\right)\right]|\xi|^{\alpha},
$$

where $\operatorname{sgn}(x)$ is the signum function.

Application of the Fourier transform to problem (1.1) with respect to the variable $x$ yields the following problem in the frequency space:

$$
\begin{cases}\frac{\partial \hat{u}(\xi, t)}{\partial t}=\gamma_{\alpha}(\xi) \hat{u}(\xi, t)+\hat{f}(\xi), & \xi \in \mathbb{R}, 0<t<T, \\ \hat{u}(\xi, 0)=0, \quad \hat{u}(\xi, T)=\hat{g}(\xi), \quad \xi \in \mathbb{R},\end{cases}
$$

where

$$
\gamma_{\alpha}(\xi)=\kappa_{\alpha} \cos \left(\frac{\pi \alpha}{2}\right)|\xi|^{\alpha}+i\left[\kappa_{\alpha}(p-q) \sin \left( \pm \frac{\pi \alpha}{2}\right)|\xi|^{\alpha}\right] .
$$

It is easy to see that the solution of problem $(2.2)$ is

$$
\hat{u}(\xi, t)=\frac{e^{\gamma_{\alpha}(\xi) t}-1}{\gamma_{\alpha}(\xi)} \hat{f}(\xi) .
$$

So we get [18] $\hat{f}(\xi)=\lambda_{\alpha}(\xi) \hat{g}(\xi)$, where

$$
\lambda_{\alpha}(\xi)=\frac{\gamma_{\alpha}(\xi)}{e^{\gamma_{\alpha}(\xi) T}-1},
$$

or equivalently

$$
f=\mathcal{F}^{-1}\left[\lambda_{\alpha}(\xi) \hat{g}(\xi)\right]=\frac{1}{\sqrt{2 \pi}} \int_{\mathbb{R}} \frac{\gamma_{\alpha}(\xi)}{e^{\gamma_{\alpha}(\xi) T}-1} \hat{g}(\xi) e^{\mathrm{i} \xi x} d \xi=: \mathcal{T} g .
$$


Obviously, the data $\hat{g}$ must decay faster than the rate $|\xi|^{-\alpha}$. However, in the practical applications, the data $g(x)$ is often obtained on the basis of reading of a physical instrument which is denoted by $g^{\delta}(x)$. In this case, such a decay is not likely to occur in the Fourier transform of the measured noisy data $g^{\delta}$. So $\mathcal{T} g^{\delta}$ can not give a reliable approximation for $f$. In the following, a truncation method based on expanded Hermite functions is introduced to reconstruct a new function $\varphi^{\delta}$ from the perturbed data $g^{\delta} . T \varphi^{\delta}$ will give a reliable approximation of $f$.

We first recall the definition of the Hermite functions and their well-known properties which are subsequently used. The normalized Hermite functions $h_{\ell}(x)$ are defined by

$$
h_{\ell}(x)=\left(2^{\ell} \ell ! \sqrt{\pi}\right)^{-1 / 2} e^{-\frac{1}{2} x^{2}} H_{\ell}(x),
$$

where the $H_{\ell}(x)$ are the usual Hermite polynomials [17]. The set of Hermite functions is the $L^{2}(\mathbb{R})$-orthogonal system [16], i.e.,

$$
\int_{\mathbb{R}} h_{\ell}(x) h_{m}(x) d x=\delta_{\ell, m},
$$

where $\delta_{\ell, m}$ is the Dirac delta function. For any $\phi \in L^{2}(\mathbb{R})$, we may write $\phi(x)=\sum_{\ell=0}^{\infty} \phi_{\ell} h_{\ell}(x)$, where

$$
\phi_{\ell}=\int_{\mathbb{R}} \phi(x) h_{\ell}(x) d x, \quad \ell=0,1,2 \ldots
$$

Let $N$ be any positive integer and

$$
\mathcal{H}_{N}=\operatorname{span}\left\{h_{0}(x), h_{1}(x), \ldots, h_{N}(x)\right\} .
$$

We shall use $c$ and $c_{\alpha}$ to denote two generic positive constants independent of any functions and the parameter $N$, but whose values may vary in each occurrence.

Lemma 1. [7] For any $\varphi \in \mathcal{H}_{N}$ and $r \geq 0$,

$$
\|\varphi\|_{r} \leq c N^{\frac{r}{2}}\|\varphi\|
$$

The $L^{2}(\mathbb{R})$-orthogonal projection $\mathcal{P}_{N}: L^{2}(\mathbb{R}) \rightarrow \mathcal{H}_{N}$ is a mapping such that, for any $\phi \in L^{2}(\mathbb{R})$,

$$
\left\langle\phi-\mathcal{P}_{N} \phi, \varphi\right\rangle=0, \quad \forall \varphi \in \mathcal{H}_{N},
$$

or equivalently,

$$
\left(\mathcal{P}_{N} \phi\right)(x)=\sum_{l=0}^{N} \phi_{\ell} h_{\ell}(x)
$$

We define also

$$
\mathcal{A} g(x)=\mathcal{D}_{x} g(x)+x g(x) .
$$


Analogously to [7], we introduce the space $\mathbb{H}_{\mathcal{A}}^{m}(\mathbb{R})$ defined by

$$
\mathbb{H}_{\mathcal{A}}^{m}(\mathbb{R})=\left\{v \mid v \text { is measurable on } \mathbb{R} \text { and }\|v\|_{m, \mathcal{A}}<\infty\right\}
$$

and equipped with the norm $\|v\|_{m, \mathcal{A}}=\left\|\mathcal{A}^{m} v\right\|$. For any $r>0$, the space $\mathbb{H}_{\mathcal{A}}^{r}(\mathbb{R})$ and its norm are defined by space interpolation [1].

Lemma 2. [7] For any $g \in \mathbb{H}_{\mathcal{A}}^{r}(\mathbb{R})$ and $0 \leq \mu \leq r$,

$$
\left\|g-\mathcal{P}_{N} g\right\|_{\mu} \leq c N^{\frac{\mu-r}{2}}\|g\|_{r, \mathcal{A}} .
$$

Lemma 3. [17] The Hermite functions are eigenfunctions of the Fourier transform

$$
\hat{h}_{\ell}(\xi)=(-i)^{\ell} h_{\ell}(\xi), \quad \ell=1,2, \ldots
$$

Suppose that the exact data $g$ and the measured data $g^{\delta}$ satisfy

$$
\left\|g^{\delta}-g\right\| \leq \delta, \quad\left\|g^{\delta}\right\|>\tau \delta
$$

where $\delta>0$ is a given constant called the error level and $\tau>1$. In this case, the function $\varphi^{n, \delta}$ can be given as

$$
g^{\delta} \rightarrow \varphi^{n, \delta}=\mathcal{P}_{n} g^{\delta}=\sum_{l=0}^{n} g_{\ell}^{\delta} h_{\ell}(x),
$$

where $g_{\ell}^{\delta}$ are produced using (2.3). The truncation parameter $n$ plays an essential role in the accuracy of these approximations. In this paper, we make use of a discrepancy principle [20] as follows to choose an $n\left(\delta, g^{\delta}\right)$ a posteriori such that:

$$
\left\|\left(I-\mathcal{P}_{n}\right) g^{\delta}\right\| \leq \tau \delta<\left\|\left(I-\mathcal{P}_{n-1}\right) g^{\delta}\right\| .
$$

Then from Lemma 3

$$
f^{n, \delta}(x):=\mathcal{T} \varphi^{n, \delta}=\mathcal{F}^{-1}\left[\lambda(\xi) \hat{\varphi}^{n, \delta}(\xi)\right]=\mathcal{F}^{-1}\left[\lambda(\xi) \sum_{\ell=0}^{n} g_{\ell}^{\delta}(-i)^{\ell} \hat{h}_{\ell}(\xi)\right]
$$

is used as an approximation of $f(x)$.

\section{Error Estimate of the Method}

In this section, the error estimate of the truncation regularization method is derived. First we give some auxiliary results.

Lemma 4. [18] For $1<\alpha<2$, there holds

$$
\left|\lambda_{\alpha}(\xi)\right| \leq c_{\alpha}\left(1+|\xi|^{2}\right)^{\frac{\alpha}{2}} .
$$

Lemma 5. For any $\varphi \in \mathcal{H}_{N}$ and $r \geq 0$,

$$
\|\mathcal{T} \varphi\|_{r} \leq c_{\alpha} N^{\frac{r+\alpha}{2}}\|\varphi\| .
$$


A Truncation Method Based on Hermite Expansion for Unknown Source 435

Proof. From Lemmas 1 and 4 and noting the fact that $\widehat{\mathcal{T} \varphi}=\lambda_{\alpha} \hat{\varphi}$,

$$
\begin{aligned}
\|\mathcal{T} \varphi\|_{r} & =\left(\int_{\mathbb{R}}\left(1+\xi^{2}\right)^{r}\left|\lambda_{\alpha}(\xi) \hat{\varphi}(\xi)\right|^{2} d \xi\right)^{1 / 2} \\
& \leq c_{\alpha}\left(\int_{\mathbb{R}}\left(1+\xi^{2}\right)^{r+\alpha}|\hat{\varphi}(\xi)|^{2} d \xi\right)^{1 / 2}=c_{\alpha}\|\varphi\|_{r+\alpha} \leq c N^{\frac{r+\alpha}{2}}\|\varphi\| .
\end{aligned}
$$

Lemma 6. Given an $r \geq 0$, for any $g \in \mathbb{H}_{\mathcal{A}}^{r+\alpha}(\mathbb{R})$ and $0 \leq \mu \leq r$,

$$
\left\|\mathcal{T}\left(I-\mathcal{P}_{N}\right) g\right\|_{\mu} \leq c_{\alpha} N^{\frac{\mu-r}{2}}\|g\|_{r+\alpha, \mathcal{A}}
$$

Proof. From Lemmas 2 and 4

$$
\begin{aligned}
& \left\|\mathcal{T}\left(I-\mathcal{P}_{N}\right) g\right\|_{\mu}=\int_{\mathbb{R}}\left(1+|\xi|^{2}\right)^{\mu}\left|\lambda_{\alpha}(\xi)\left(\mathcal{F}\left(I-\mathcal{P}_{N}\right) g\right)(\xi)\right|^{2} d \xi \\
& \quad \leq c_{\alpha}\left\|\left(I-\mathcal{P}_{N}\right) g\right\|_{\mu+\alpha} \leq c_{\alpha} N^{\frac{\mu+\alpha-r+\alpha}{2}}\|g\|_{r+\alpha, \mathcal{A}} \leq c_{\alpha} N^{\frac{\mu-r}{2}}\|g\|_{r+\alpha, \mathcal{A}} .
\end{aligned}
$$

Lemma 7. If $\|\mathcal{T} \varphi\|_{r}$ is bounded, then we have

$$
\|\mathcal{T} \varphi\| \leq\left(c_{\alpha}\|\varphi\|\right)^{\frac{r}{r+\alpha}}\|\mathcal{T} \varphi\|_{r}^{\frac{\alpha}{r+\alpha}} .
$$

Proof. By the Hölder inequality, Lemma 4 and noting that $\widehat{\mathcal{T} \varphi}=\lambda_{\alpha} \hat{\varphi}$

$$
\begin{aligned}
\|\mathcal{T} \varphi\|^{2} & =\int_{\mathbb{R}}\left|\lambda_{\alpha}(\xi)\right|^{2}|\hat{\varphi}|^{2} d \xi=\int_{\mathbb{R}}\left|\lambda_{\alpha}(\xi)\right|^{2}|\hat{\varphi}|^{\frac{2 \alpha}{r+\alpha}}|\hat{\varphi}|^{\frac{2 r}{r+\alpha}} d \xi \\
& \leq\left[\int_{\mathbb{R}}\left(\left|\lambda_{\alpha}(\xi)\right|^{2}|\hat{\varphi}|^{\frac{2 \alpha}{r+\alpha}}\right)^{\frac{r+\alpha}{\alpha}} d \xi\right]^{\frac{\alpha}{r+\alpha}}\left[\int_{\mathbb{R}}\left(|\hat{\varphi}|^{\frac{2 r}{r+\alpha}}\right)^{\frac{r+\alpha}{r}} d \xi\right]^{\frac{r}{r+\alpha}} \\
& \leq\left[c_{\alpha}^{\frac{2 r}{\alpha}} \int_{\mathbb{R}}\left(1+\xi^{2}\right)^{r}|\widehat{\mathcal{T} \varphi}|^{2} d \xi\right]^{\frac{\alpha}{r+\alpha}}\|\varphi\|^{\frac{2 r}{r+\alpha}}=c_{\alpha}^{\frac{2 r}{r+\alpha}}\|\varphi\|^{\frac{2 r}{r+\alpha}}\|\mathcal{T} \varphi\|_{r}^{\frac{2 \alpha}{r+\alpha}}
\end{aligned}
$$

Theorem 1. Suppose that the condition (2.4) holds for some $g$ and $g^{\delta}, g \in$ $H_{\mathcal{A}}^{r+\alpha}(\mathbb{R}) . f^{n, \delta}(x)$ is defined by $(2.6)$ and $(2.7)$, then

$$
\left\|f^{n, \delta}-f\right\|=O\left(\delta^{\frac{r}{r+\alpha}}\right) .
$$

Proof. By Lemmas 5 and 6,

$$
\begin{gathered}
\left\|f^{n, \delta}-f\right\|_{r}=\left\|\mathcal{T} \varphi^{n, \delta}-\mathcal{T} g\right\|_{r}=\left\|\mathcal{T} \mathcal{P}_{n} g^{\delta}-\mathcal{T} \mathcal{P}_{n} g+\mathcal{T} \mathcal{P}_{n} g-\mathcal{T} g\right\|_{r} \\
\leq\left\|\mathcal{T} \mathcal{P}_{n}\left(g^{\delta}-g\right)\right\|_{r}+\left\|\mathcal{T}\left(I-\mathcal{P}_{n}\right) g\right\|_{r} \leq c n^{\frac{r+\alpha}{2}} \delta+c\|g\|_{r+\alpha, \mathcal{A}}
\end{gathered}
$$

On the other hand,

$$
\begin{aligned}
\left\|\mathcal{P}_{n-1} g-g\right\| & =\left\|\left(\mathcal{P}_{n-1} g^{\delta}-g^{\delta}\right)-\left(I-\mathcal{P}_{n-1}\right)\left(g-g^{\delta}\right)\right\| \\
& \geq\left\|\mathcal{P}_{n-1} g^{\delta}-g^{\delta}\right\|-\left\|\left(I-\mathcal{P}_{n-1}\right)\left(g-g^{\delta}\right)\right\| \|
\end{aligned}
$$

From (2.6), we have $\left\|\mathcal{P}_{n-1} g^{\delta}-g^{\delta}\right\|>\tau \delta$. And from (2.4)

$$
\left\|\left(I-\mathcal{P}_{n-1}\right)\left(g-g^{\delta}\right)\right\| \leq\left\|\left(g-g^{\delta}\right)\right\| \leq \delta .
$$


So $\left\|\mathcal{P}_{n-1} g-g\right\| \geq(\tau-1) \delta$. From Lemma 2

$$
(\tau-1) \delta \leq\left\|\mathcal{P}_{n-1} g-g\right\| \leq c(n-1)^{-\frac{r+\alpha}{2}}\|g\|_{r+\alpha, \mathcal{A}} .
$$

Thus we can get

$$
n \leq\left(\frac{c\|g\|_{r+\alpha, A}}{\tau-1}\right)^{\frac{2}{r+\alpha}} \delta^{-\frac{2}{r+\alpha}}+1 .
$$

By (3.1) and (3.2), there exists an bounded value $M_{\alpha, \delta}$ such that

$$
\left\|f^{n, \delta}-f\right\|_{r}=\left\|\mathcal{T}\left(\varphi^{n, \delta}-g\right)\right\|_{r} \leq M_{\alpha, \delta} .
$$

Moreover,

$$
\left\|\varphi^{n, \delta}-g\right\| \leq\left\|\varphi^{n, \delta}-g^{\delta}\right\|+\left\|g^{\delta}-g\right\| \leq(\tau+1) \delta .
$$

The assertion of theorem can be obtained by (3.3), (3.4) and Lemma 7 .

\section{Numerical Tests}

We first discuss the algorithm implementation, and then some numerical tests is presented to verify the effectiveness of the method.

To reduce the impact of discretization errors, the discretization knots are chosen as $x_{j}=\sigma_{N, j}, j=0,1, \ldots, N$, where $\sigma_{N, j}(0 \leq j \leq N)$ are the zeros of $H_{N+1}(x)$. We first get the data $g=\left\{g\left(x_{j}\right)\right\}_{j=0}^{N}$ representing values of $g(x)$ on the knots, then obtain the perturbation data $g^{\delta}$ as follows

$$
g^{\delta}\left(x_{i}\right)=g\left(x_{i}\right)+\epsilon_{i}, \quad\left|\epsilon_{i}\right|<\delta_{1},
$$

where $\{\epsilon\}_{i=0}^{N}$ are generated by $(2 \times \operatorname{rand}(N+1,1)-1) \times \delta_{1}$ in Matlab.

Let $\omega_{N, j}$ be the corresponding Hermite-Gauss weights, namely

$$
\omega_{N, j}=\rho_{N, j} e^{\sigma_{N, j}^{2}},
$$

where $\rho_{N, j}$ are the Christoffel numbers of the stand Hermite-Gauss interpolation (see, for instance, [8]). Analogously to the method in Ref. [23], we can get $\varphi^{n, \delta}$ by using Hermite-pseudospectral method with the discrete inner product and the discrete norm

$$
\langle u, v\rangle_{N}=\sum_{j=0}^{N} u\left(\sigma_{N, j}\right) v\left(\sigma_{N, j}\right) \omega_{N, j}, \quad\|v\|_{N}=\langle v, v\rangle_{N}^{1 / 2} .
$$

Usually it is difficult to get the analytic solution of the fractional partial differential equation (1.1) with some boundary conditions, so we have to obtain the data $g$ by numerical methods for a given $f(x)$ at first. The numerical procedure is described as follows:

Step 1: Calculating the Hermite interpolation of $f$

$$
\left(\mathcal{I}_{N} f\right)(x)=\sum_{j=0}^{N} \mathbf{f}_{j} h_{j}(x),
$$


where $\mathbf{f}_{j}=\left\langle f, h_{j}\right\rangle_{N}$.

Step 2: Obtaining an approximation of the Fourier transform of $g$

$$
\hat{g}(\xi)=\frac{1}{\lambda_{\alpha}(\xi)} \hat{f}(\xi) \approx \frac{1}{\lambda_{\alpha}(\xi)} \sum_{j=0}^{N}(-i)^{j} \mathbf{f}_{j} h_{j}(\xi) .
$$

Step 3: Computing the values

$$
g\left(x_{j}\right)=\frac{1}{\sqrt{2 \pi}} \int_{\mathbb{R}} \hat{g}(\xi) e^{\mathrm{i} \xi x_{j}} d \xi .
$$

In the following, we present numerical results of two examples to check the effectiveness of the method. The accuracy of results are measured by

$$
E_{f}=\left\|f^{n, \delta}-f\right\|_{N}=\left[\sum_{j=0}^{N} \omega_{N, j}\left(f^{n, \delta}\left(x_{j}\right)-f\left(x_{j}\right)\right)^{2}\right]^{1 / 2} .
$$

For simplicity, all examples are computed by using Matlab with $N=256$, $\kappa_{\alpha}=1, p=q=0.5$ and $T=1$ (If we take $N, \kappa_{\alpha}, p, q$ and $T$ with other values, we can also obtain a satisfactory result).

$$
E_{f}=\left\|f^{n, \delta}-f\right\|_{N}=\left[\sum_{j=0}^{N} \omega_{N, j}\left(f^{n, \delta}\left(x_{j}\right)-f\left(x_{j}\right)\right)^{2}\right]^{1 / 2} .
$$

Example 1. [18] First we consider the function

$$
f(x)=\left(\frac{x^{3}}{4}-\frac{3 x}{2}\right) e^{-\frac{x^{2}}{4}} .
$$

Fig. 1 shows the error of the exact solution $f$ and the regularization solution $f^{n, \delta}$ for $\alpha=1.1,1.5,1.9$.

Figs. 2-3 exhibit the variation of $E_{f}$ with the changes of $\alpha$ and $\delta_{1}$, respectively.

Table 1. $E_{f}$ for various $\alpha$ and $\delta$.

\begin{tabular}{cccc}
\hline$\delta_{1}$ & $\alpha=1.1$ & $\alpha=1.5$ & $\alpha=1.9$ \\
\hline $1 \mathrm{e}-1$ & 0.0181 & 0.0366 & 0.0537 \\
$1 \mathrm{e}-2$ & 0.0022 & 0.0072 & 0.0131 \\
$1 \mathrm{e}-3$ & $2.41 \mathrm{e}-4$ & 0.0014 & 0.0023 \\
$1 \mathrm{e}-4$ & $4.04 \mathrm{e}-5$ & $2.36 \mathrm{e}-4$ & $3.91 \mathrm{e}-4$ \\
\hline
\end{tabular}

Table 1 lists error for different noise level $\delta_{1}$. We can see that when $\delta_{1}$ decreases from 0.1 to 0.0001 , the relative errors become smaller. 


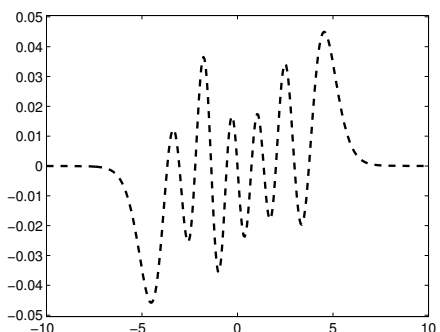

(a) $\delta_{1}=0.1, \alpha=1.1$

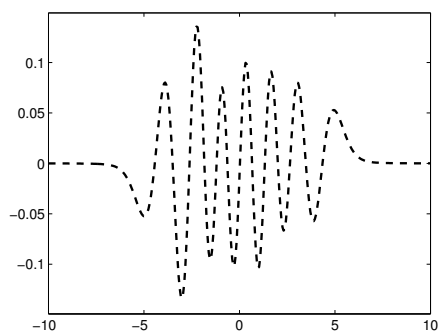

(c) $\delta_{1}=0.1, \alpha=1.5$.

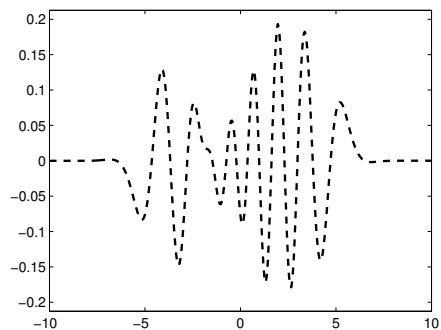

(e) $\delta_{1}=0.1, \alpha=1.9$.

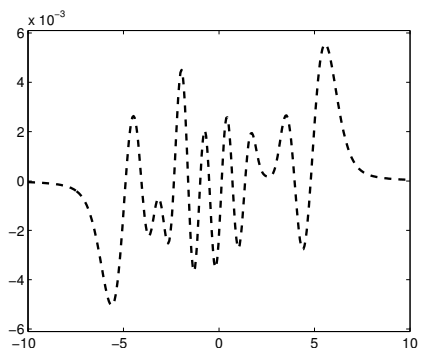

(b) $\delta_{1}=0.01, \alpha=1.1$.

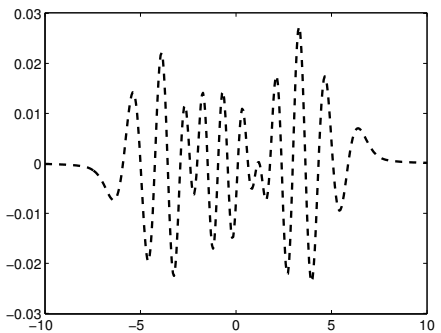

(d) $\delta_{1}=0.01, \alpha=1.5$.

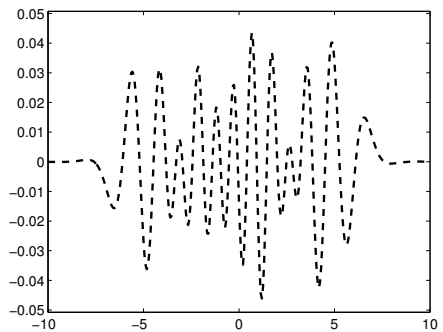

(f) $\delta_{1}=0.01, \alpha=1.9$.

Figure 1. The error of the exact solution and its approximation (Example 1).

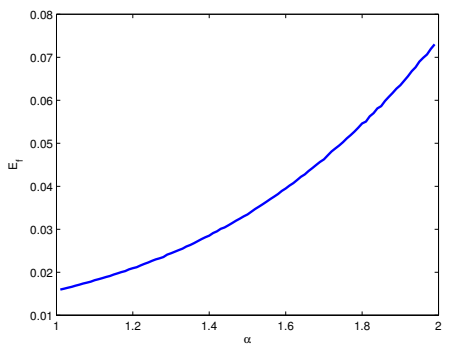

(a) $\delta_{1}=0.1$

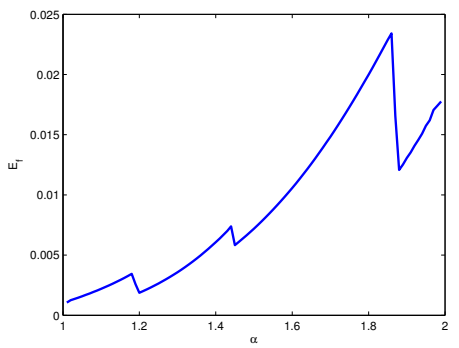

(b) $\delta_{1}=0.01$

Figure 2. The variation of $E_{f}$ with $\alpha$ (Example 1). 


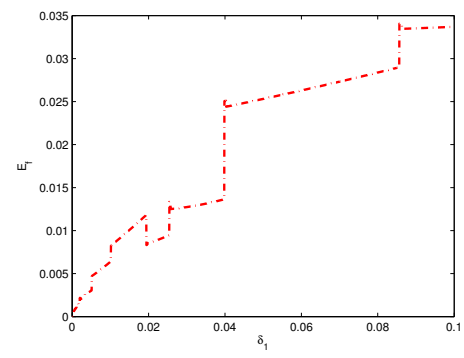

(a) $\alpha=1.5$

Figure 3. The variation of $E_{f}$ with $\delta_{1}$ (Example 1$)$.

Example 2. Consider a non-smooth

$$
f(x)=\left\{\begin{array}{lc}
x+8, & -8 \leq x \leq-4 \\
-x, & -4<x \leq 4 \\
x-8, & 4<x \leq 8 \\
0, & \text { otherwise }
\end{array}\right.
$$

The errors are given in Table 2 and the error of the exact and computed functions are shown in Fig. 4.

Table 2. $E_{f}$ for various $\alpha$ and $\delta$.

\begin{tabular}{cccc}
\hline$\delta_{1}$ & $\alpha=1.1$ & $\alpha=1.5$ & $\alpha=1.9$ \\
\hline $1 \mathrm{e}-1$ & 0.0271 & 0.1125 & 0.3106 \\
$1 \mathrm{e}-2$ & 0.0066 & 0.0278 & 0.0674 \\
$1 \mathrm{e}-3$ & 0.0026 & 0.0063 & 0.0179 \\
$1 \mathrm{e}-4$ & 0.0016 & 0.0027 & 0.0092 \\
\hline
\end{tabular}

The variation of $E_{f}$ with the changes of $\alpha$ and $\delta_{1}$ are exhibited in Figs. $5-6$, respectively. It is easy to see that the numerical effect of the method is satisfactory. The numbers in Table 2 show that the convergence rate is slowly than the one in Table 1, this accords with our theoretical results.

These examples demonstrate the potential effectiveness of our approximation scheme, for suitable problems.

\section{Conclusion}

We have proposed an effective numerical method for determining unknown source in a space fractional diffusion equation. We have proved that the numerical method is stable and an error estimate has been obtained. Numerical tests indicate that the proposed method works well. 


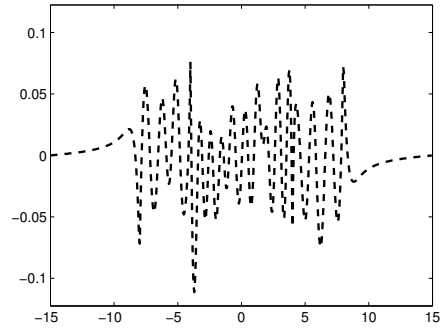

(a) $\delta_{1}=0.1, \alpha=1.1$.

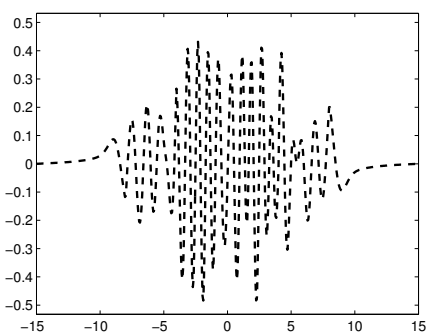

(c) $\delta_{1}=0.1, \alpha=1.5$.

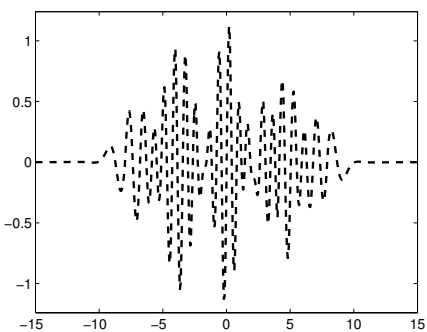

(e) $\delta_{1}=0.1, \alpha=1.9$.

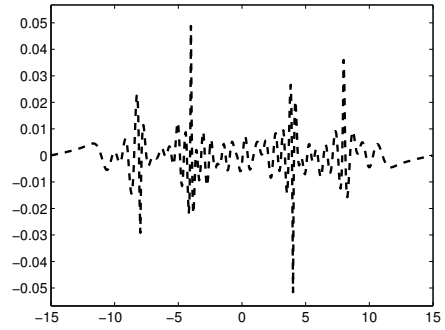

(b) $\delta_{1}=0.01, \alpha=1.1$.

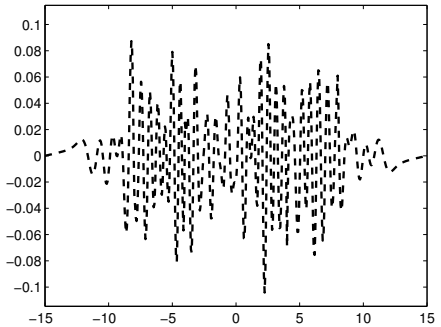

(d) $\delta_{1}=0.01, \alpha=1.5$.

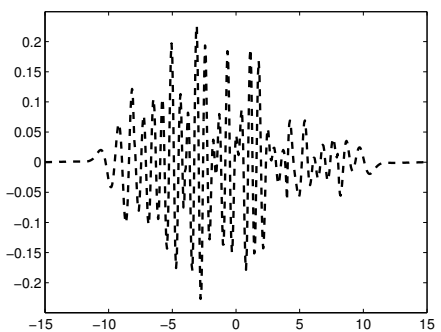

(f) $\delta_{1}=0.01, \alpha=1.9$.

Figure 4. The error of the exact solution and its approximation (Example 2).

\section{Acknowledgments}

The authors thank the referees for their kind suggestions which have improved our paper very much. The project is supported by the National Natural Science Foundation of China (No. 11201085).

\section{References}

[1] R.A. Adams. Sobolev Spaces. Pure Appl. Math. Academic Press, New York, 1975.

[2] F. Berntsson. A spectral method for solving the sideways heat equation. Inverse Problems, 15(4):891-906, 1999. http://dx.doi.org/10.1088/0266$5611 / 15 / 4 / 305$. 


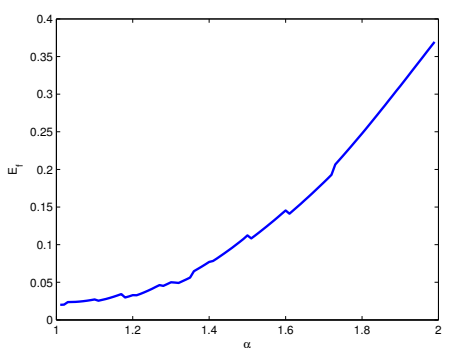

(a) $\delta_{1}=0.1$

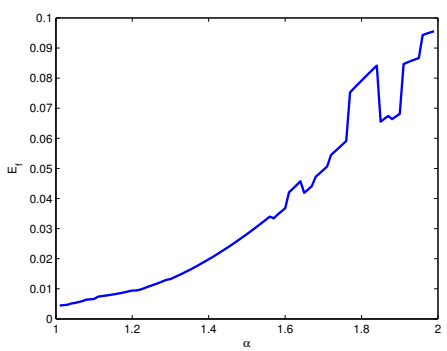

(b) $\delta_{1}=0.01$

Figure 5. The variation of $E_{f}$ with $\alpha$ (Example 2).

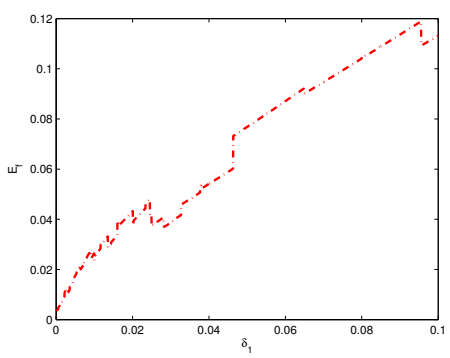

(a) $\alpha=1.5$

Figure 6. The variation of $E_{f}$ with $\delta_{1}$ (Example 2).

[3] A.N. Bondarenko and D.S. Ivaschenko. Numerical methods for solving inverse problems for time fractional diffusion equation with variable coefficient. J. Inverse Ill-Posed Probl., 17(5):419-440, 2009.

http://dx.doi.org/10.1515/JIIP.2009.028.

[4] J. Cheng, J. Nakagawa, M. Yamamoto and T. Yamazaki. Uniqueness in an inverse problem for a one-dimensional fractional diffusion equation. Inverse Problems, 25(11):115002, 2009. http://dx.doi.org/10.1088/0266$5611 / 25 / 11 / 115002$.

[5] F.F. Dou, C.L. Fu and F.L. Yang. Optimal error bound and fourier regularization for identifying an unknown source in the heat equation. J. Comput. Appl. Math., 230(2):728-737, 2009. http://dx.doi.org/10.1016/j.cam.2009.01.008.

[6] L. Eldén, F. Berntsson and T. Reginska. Wavelet and fourier methods for solving the sideways heat equation. SIAM J. Sci. Comput., 21(6):2187-2205, 2000. http://dx.doi.org/10.1137/S1064827597331394.

[7] B.Y. Guo, J. Shen and C.L. Xu. Spectral and pseudospectral approximations using hermite functions: application to the dirac equation. Adv. Comput. Math., 19(1):35-55, 2003. http://dx.doi.org/10.1023/A:1022892132249.

[8] B.Y. Guo and C.L. Xu. Hermite pseudospectral method for nonlinear partial differential equations. ESAIM: Math. Model. Numer. Anal., 34(04):859-872, 2000. http://dx.doi.org/10.1051/m2an:2000100. 
[9] G.A. Kriegsmann and W.E. Olmstead. Source identification for the heat equation. Appl. Math. Lett., 1(3):241-245, 1988. http://dx.doi.org/10.1016/0893-9659(88)90084-5.

[10] J.J. Liu and M. Yamamoto. A backward problem for the time-fractional diffusion equation. Appl. Anal., 89(11):1769-1788, 2010. http://dx.doi.org/10.1080/00036810903479731.

[11] R. Metzler and J. Klafter. The random walk's guide to anomalous diffusion: a fractional dynamics approach. Phys. Rep., 339(1):1-77, 2000.

http://dx.doi.org/10.1016/S0370-1573(00)00070-3.

[12] R. Metzler and J. Klafter. The restaurant at the end of the random walk: recent developments in the description of anomalous transport by fractional dynamics. J. Phys. A, 37(31):R161, 2004. http://dx.doi.org/10.1088/0305-4470/37/31/R01.

[13] D.A. Murio. Stable numerical solution of a fractional-diffusion inverse heat conduction problem. Comput. Math. Appl., 53(10):1492-1501, 2007. http://dx.doi.org/10.1016/j.camwa.2006.05.027.

[14] C. Piret and E. Hanert. A radial basis functions method for fractional diffusion equations. J. Comput. Phys., 238:71-81, 2013.

http://dx.doi.org/10.1016/j.jcp.2012.10.041.

[15] I. Podlubny. Fractional Differential Equations. Academic Press, San Diego, 1999.

[16] G. Szegö. Orthogonal Polynomials. Amer. Math. Soc., 1975.

[17] S. Thangavelu. Lectures on Hermite and Laguerre Expansions. Princeton University Press, Princeton, 1993.

[18] W.Y. Tian, C. Li, W.H. Deng and Y.J. Wu. Regularization methods for unknown source in space fractional diffusion equation. Math. Comput. Simulation, 85:4556, 2012. http://dx.doi.org/10.1016/j.matcom.2012.08.011.

[19] W.Y. Tian, H. Zhou and W.H. Deng. A class of second order difference approximation for solving space fractional diffusion equations, 2012. arXiv preprint, arXiv: 1201.5949

[20] G.M. Vainikko. The discrepancy principle for a class of regularization methods. USSR Comput. Math. Math. Phys., 22(3):1-19, 1982. http://dx.doi.org/10.1016/0041-5553(82)90120-3.

[21] X.T. Xiong and C.L. Fu. Determining surface temperature and heat flux by a wavelet dual least squares method. J. Comput. Appl. Math., 201(1):198-207, 2007. http://dx.doi.org/10.1016/j.cam.2006.02.014.

[22] Z.Y. Zhao. Numerical analytic continuation by a mollification method based on hermite function expansion. Inverse Problems, 28(4):045002, 2012. http://dx.doi.org/10.1088/0266-5611/28/4/045002.

[23] Z.Y. Zhao and J.F. Liu. Hermite spectral and pseudospectral methods for numerical differentiation. Appl. Numer. Math., 61(12):1322-1330, 2011. http://dx.doi.org/10.1016/j.apnum.2011.09.006.

[24] H. Zhou, W.Y. Tian and W.H. Deng. Quasi-compact finite difference schemes for space fractional diffusion equations. J. Sci. Comput., 56(1):1-22, 2013. http://dx.doi.org/10.1007/s10915-012-9661-0. 